\title{
An Interoperable IP based WSN for Smart Irrigation Systems
}

\author{
Md. Zainal Abedin ${ }^{1}$, Abu Sayeed Chowdhury ${ }^{1}$, Mohammad Shahadat Hossain ${ }^{2}$, \\ Karl Andersson ${ }^{3}$, and Razuan Karim ${ }^{1}$

\begin{abstract}
${ }^{1}$ Faculty of Science, Engineering and Technology, University of Science \& Technology, Chittagong-1079, Bangladesh ${ }^{2}$ Department of Computer Science and Engineering, University of Chittagong-4331, Bangladesh

${ }^{3}$ Luleå University of Technology, SE-931 87 Skellefteå, Sweden

jakcse99@gmail.com, chowdhurysayed@yahoo.com, hossain_ms@cu.ac.bd, karl.andersson@ltu.se, karim7@usa.com
\end{abstract}

\begin{abstract}
Wireless Sensor Networks (WSN) have been highly developed which can be used in agriculture to enable optimal irrigation scheduling. Since there is an absence of widely used available methods to support effective agriculture practice in different weather conditions, WSN technology can be used to optimise irrigation in the crop fields. This paper presents architecture of an irrigation system by incorporating interoperable IP based WSN, which uses the protocol stacks and standard of the Internet of Things paradigm. The performance of fundamental issues of this network is emulated in Tmote Sky for 6LoWPAN over IEEE 802.15.4 radio link using the Contiki $O S$ and the Cooja simulator. The simulated results of the performance of the WSN architecture presents the Round Trip Time (RTT) as well as the packet loss of different packet size. In addition, the average power consumption and the radio duty cycle of the sensors are studied. This will facilitate the deployment of a scalable and interoperable multi hop WSN, positioning of border router and to manage power consumption of the sensors.
\end{abstract}

Keywords-6LoWPAN, IEEE 802.15.4, Irrigation, Internet of Things, Contiki; Cooja, Round Trip Time, Radio Duty Cycle

\section{INTRODUCTION}

Shortage of fresh water is a common problem in the world. The demand for water supplies is expected to increase due to population growth; food demand needs, and changes in water availability. Irrigation is a primary user of freshwater, with the Food and Agricultural Organization of the United Nations (FAO, 2014) reporting that $70 \%$ of freshwater in the world is used for irrigation purposes. Irrigation is expected to be much more crucial for the food production in the near future, as most probable climate change scenarios predict large water deficits in certain areas in the world. Irrigation holds the most promise for increasing food productivity and security, provided it is managed efficiently. Also irrigation consumes large quantities of energy, which is a crucial constraint for development. To better manage the competing demands for water, agricultural policies will have to make water efficiency a priority. Hence the scientific and technology based irrigation scheduling of water is one of the ways to improve the irrigation processes to optimize the use of water and energy consumption. The incorporation of sensors in the context of agricultural production for water management and conservation has concerned an increasing interest for establishing irrigation management strategies. The use of water for agricultural production in water scarcity regions requires innovative and sustainable research and an appropriate transfer of technologies. Moreover, food security is an important issue since systems aiming at ensuring optimal production of crops form a vital part of any country's overall security system.

Ubiquitous and pervasive computing are key technologies significantly contributing to the advent of IoT. They bring computation capability to physical objects, which can communicate, in the Internet by producing, consuming, and computing information. WSNs are a prevalent instance of ubiquitous computing that enables small things to connect to the Internet. Sensor networks today are sensing and computing environmental phenomena and thus such a WSN can be used in real time application like irrigation scheduling. In reality these types of networks are heterogeneous by nature, arises from the fact that there are different types of WSN devices that do not use the same standards. The migration of WSN communication towards an all-IP mode, eliminate most of the heterogeneity. The Internet is smoothly migrating from an Internet of people towards an Internet of Things (IoT). Such a migration induces a strong level of complexity when handling interoperability between the heterogeneous IoT and also these types of networks are low power and lossy network. To support the connectivity, interoperability ad compatibility of heterogeneous WSN, IPv6 over Low Power Wireless Personal Area Network (6LoWPAN) was proposed by the IETF working group.

Therefore the system proposed in this paper uses the wireless sensor networks and the architecture of Internet of things, allowing the users to analyze the moisture level of the field obtained by the array of sensors from any smart device in efficient way to take the decision of irrigation scheduling. This paper presents a multi hop WSN for smart irrigation scheduling that relies on 6LoWPAN, RPL and IEEE $802.15 .4 \mathrm{~g}$ protocols along with the performance evaluation of the WSN in terms of round trip time, packet loss and radio duty cycles of the sensors.

The remainder of this paper is organized in the following way: Section 2 covers related work, whereas Section 3 presents our proposed system architecture. Section 4 includes details on our prototype design, while Section 5 conveys information about the simulations performed. Section 6 presents our results and the analysis performed, while Section 7 finally concludes the paper and indicates future work. 


\section{RELATED WORK}

In GSM (Global System for Mobile Communication) based automated irrigation control using rain gun [1] irrigation will take place only when there will be intense requirement of water that save a large quantity of water. This system is not affordable economically due to coverage range of agriculture land. Siuli Roy et al. [2] proposed a Zigbee based wireless sensor network with the help of IEEE 802.15.4 link layer protocol. This work focuses to monitor agricultural parameter remotely and to control irrigation and fertilization. Evapotranspiration (ET) is affected by solar radiation, temperature, humidity, wind speed, and crop factors, such as stage of growth, variety and plant density, management elements, soil properties, pest, and disease control [3]. Systems based on ET have been developed that agree to water savings of up to $42 \%$ on time-based irrigation program [4]. To monitor the agricultural system a microcontroller based system developed by Zeldi [5], where the real time values of atmospheric condition displayed by the microcontroller by sensing of different parameters of agriculture like soil moisture, humidity and temperature. An electromagnetic moisture sensor was the basis for developing an irrigation system at a savings of $53 \%$ of water compared with irrigation by sprinklers in an area of $1000 \mathrm{~m} 2$ of territory [6]. By using soil sensor and an evaporimeter [7], to adjust the irrigation in different weather conditions, a scheduling system was developed to reduce the water consumption.

However no performance analysis was made to look after the power consumption and fundamental issues of 6LoWPAN protocol stack in these studies. These issues made us to decide to study the performances of irrigation system through Cooja Simulator. Since 6LoWPAN is an immerging technology and is suitable for constraint environment, smart irrigation based on this stack can be a new application area. The purpose of this research is to develop a smart irrigation system using 6LoWPAN wireless sensor network [8][9][10][11][12][13] and analyze the performances of the WSN. The system will be integrated with wireless sensor network technology where solar energy can be used to overcome the power crisis along with optimizing the irrigation management based on the 6LoWAN standard.

Our own previous work includes performance analysis of an IP based protocol stack for WSNs [14][15].

\section{SYSTEM ARCHITECTURE}

Fig. 1 depicts the systems architecture to implement real time irrigation scheduling in a crop field.

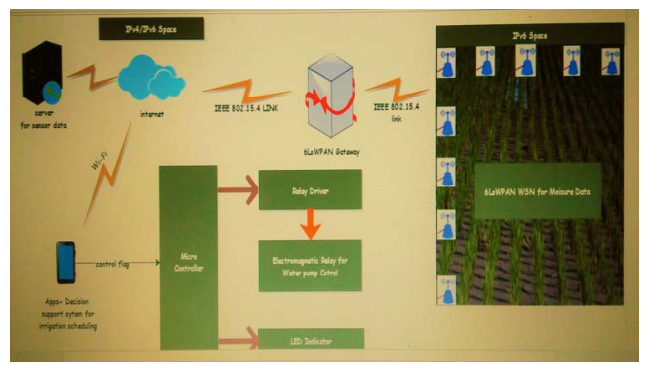

Fig. 1. Proposed system architecture
The architecture consists of a LoWPAN wireless sensor network, an IoT gateway for Internet connectivity, a web server to store sensor data, application software implementing our decision support system to provide irrigation scheduling, and a control system for interfacing and controlling the irrigation pumps.

A LoWPAN is the collection of 6LoWPAN Nodes, which share a common IPv6 address, prefix. LoWPAN are connected to other IP networks through edge routers or border router. The edge router plays an important role as it routes traffic in and out of the LoWPAN, while handling 6LoWPAN compression and neighbor discovery for the LoWPAN. Multiple edge routers can be supported in the same LoWPAN if they share a common backbone link. Each LoWPAN node is identified by a unique IPv6 address, and is capable of sending and receiving IPv6 packets. Typically LoWPAN Nodes support ICMPv6 traffic such as ping and use the user datagram protocol (UDP) as a transport protocol. In the WSN, the upper leftmost sensor node acts as edge router as well as sink node and also the root of directed acyclic graph formed by the network topology. The rest of the nodes act as source nodes and send their sensing data to the sink node, which then forwards to the destination server through the gateway. To connect the WSN with the Internet backbone, a gateway is deployed between edge router and the LoWPAN. The gateway enables the connectivity of the LoWPAN to the Internet. Hence sensor data from the radio network can be transmitted to the server located anywhere in the Internet.

The server in the architecture is used to collect and store the sensors data. The sensors sense moisture level of the crop field and forward the data to the server through the gateway. We can implement the server as a cloud from where the farmer, user or client retrieves sensor data to analysis for making decision such as moisture level, to make irrigation scheduling.

The web based decision support system (DSS) analyzes the real time moisture level of the crop field and provides users with an irrigation schedule for crops during different phases of phonological development based on moisture level. If the DSS recommends for irrigation, then the user send a positive flag to the control system which then switches on the relay driver to turn on the irrigation pump. A relay is an electromagnetic switch operated by a relatively small electric current that can switch a much larger electric device. The LED in the microcontroller indicates the ON and OFF states of the relay circuit. Here we propose the source of energy for the pump is solar renewable energy due to power crisis of Bangladesh and remote location of the crop field.

\section{PROTOTYPE DESGIN}

We have implemented a prototype of smart irrigation system. A moisture sensor is used to detect the moisture. An Arduino Uno board is used to control whole system. The Arduino module compares the moisture level of the field with the threshold moisture level. Arduino will instruct the pump to be activated if the moisture of the field is smaller than the set threshold. When the moisture level is more than the threshold, the irrigation will be stopped by the Arduino. A solar system is used to provide the power need for this system. 
Fig. 2 presents the prototype implementation of our proposed system.

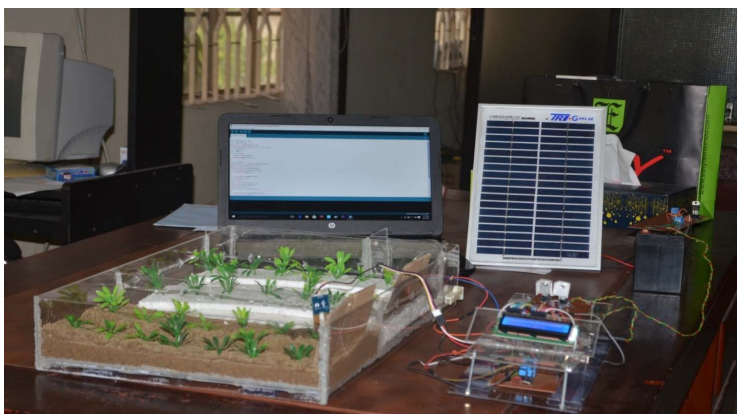

Fig. 2. Prototype Implementation of Smart Irrigation System

\section{Simulation SCENARIO}

To closely reflect real deployments, nodes run Contiki OS with the whole protocol stack. We have compiled Contiki for the Tmote Sky platform based on MSP430 microcontroller with $10 \mathrm{~KB}$ of RAM and an IEEE 802.15.4 compliant radio. By using the MSP430 hardware emulator, Cooja thus takes into account all the hardware constraints of the devices. The simulation scenario is presented in Fig. 3.

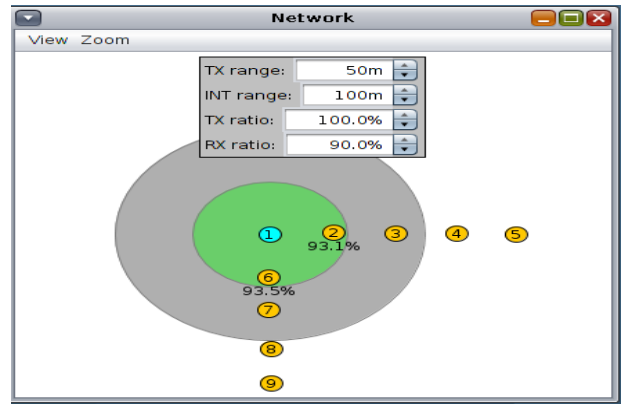

Fig. 3. WSN topology and radio environment in Cooja. The green disk represents the transmission range and the gray disk represents the interference range of the sensor. The yellow nodes are source nodes while the blue node is the sink node.

The propagation model used in the simulations is COOJA's Unit Disk Graph Medium (UDGM) with constant loss. This model uses two concentric disks of different radius. Any node within the transmission range receives transmitted packets with a probability of $100 \%$ (default value). Any node within the interference range but outside of the transmission range is not able to receive any packets but see its own transmissions affected. Finally nodes outside of the interference range are not able to receive packets and are not affected by transmitted packets. The border router or edge router is typically a device sitting at the edge of our network, which allows us to talk to outside networks using its built-in network interfaces, such as $\mathrm{Wi}-\mathrm{Fi}$, Ethernet, Serial, etc. In Contiki the current and most used border router application implements a serial based interface called SLIP, it allows to connect a given mote to a host using scripts like tunslip6 over the serial port, creating a tunneled network interface, which can be given an IPv6 prefix to set the network global IPv6 addresses. Node 1 corresponds to the border router, which has only one neighbor in its wireless coverage area the sensors are deployed to establish multi hops network from the border router. The simulation parameters including the protocol stack used are shown below in Table 1. For capturing data collected from sensors we use Collect view visualization tool provided by the Contiki OS.

TABLE 1. SimULATION PARAMETERS

\begin{tabular}{|c|c|}
\hline \multicolumn{2}{|c|}{ Test Environment } \\
\hline OS & Contiki OS \\
\hline Simulator & Cooja \\
\hline Radio Medium & Unit Disk Graph Medium Distance \\
\hline Mote Types/startup delay & T-mote Sky/ 1000 ms \\
\hline Node Transmission range & $50 \mathrm{~m}$ \\
\hline Node Carrier sensing range & $100 \mathrm{~m}$ \\
\hline TX/RX ratio & $100 \% / 90 \%$ \\
\hline \multicolumn{2}{|c|}{ Communication Protocols } \\
\hline Network & Routing (RPL), Adaption \\
& (6LoWPAN) \\
\hline Data Link & CSMA, Radio Duty Cycling (Contiki \\
& MAC) \\
\hline Physical & IEEE 802.15.4 \\
\hline \multicolumn{2}{|c|}{} \\
\hline
\end{tabular}

\section{RESULTS AND ANALYSIS}

To illustrate the performance of the proposed WSN scenario we evaluate three important parameters named round trip time as delay, packet loss and energy consumption (radio duty cycle and average power consumption. This evaluation also tests the RPL routing protocol used in 6LoWPAN, as the scenario features nodes with 1 to 4 hops to the router.

\section{A. Round Trip Time (RTT)}

The RTT is an important parameter of concern as it is an indicator of the network congestion as well as the processing delays. It is computed as function of time by the ping request as the time difference between the time of successful response for a request and the time instant at which the request is sent. In our simulation, payload size is varied from 8 bytes to 56 bytes. Twenty ICMP messages are sent to each source node in the WSN through the border router from Contiki client and the statistics of round trip time are recorded and plotted in Matlab simulation software. The plots below illustrate minimum RTT, maximum RTT, median RTT and deviations of RTT for different packet size and multi hop scenarios. It can be seen from Fig. 4 and Fig. 5 that packets moving further away from the sink experience higher RTT, as expected and with the higher payload, the RTT is also higher especially in multi hop cases. Fig. 7 presents cumulative distribution function (CDF) as a function of deviation of RTT for different hop counts, while Fig. 8 shows packet loss for different hop.

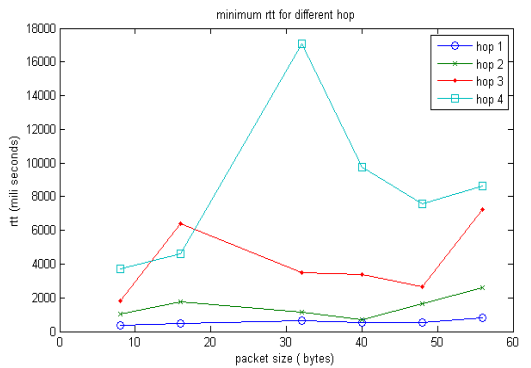

Fig. 4. Minimum RTT 


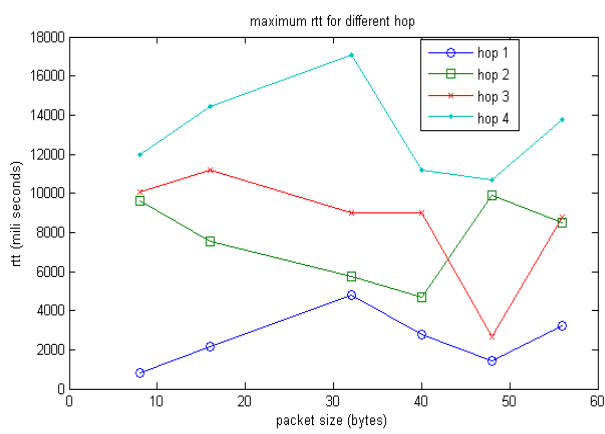

Fig. 5. Maximum RTT

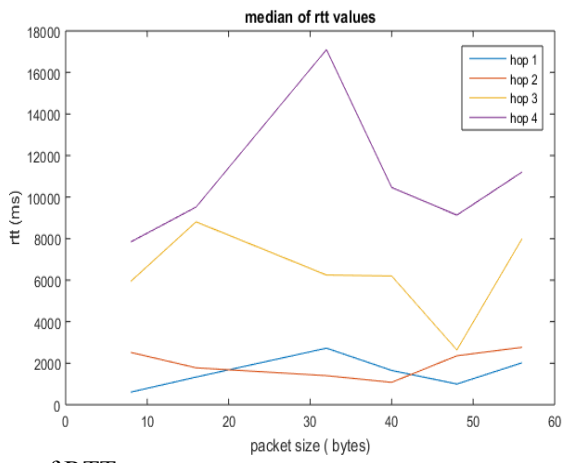

Fig. 6. Median of RTT

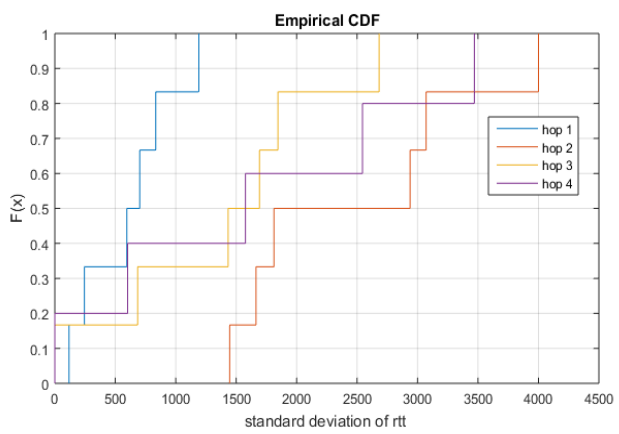

Fig. 7. CDF of Deviation of RTT for different hops

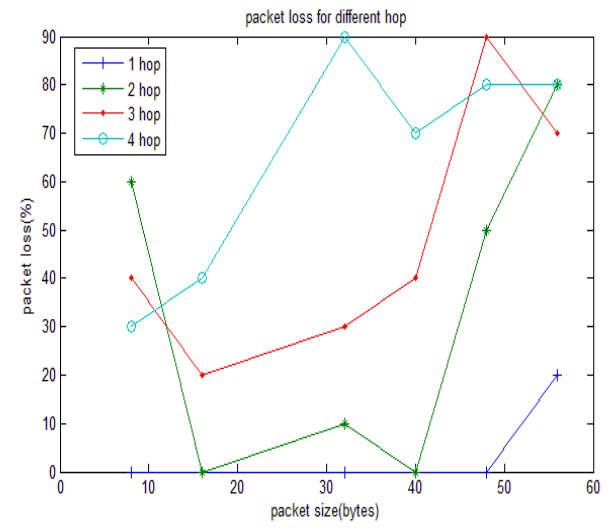

Fig. 8. Packet loss for different hop

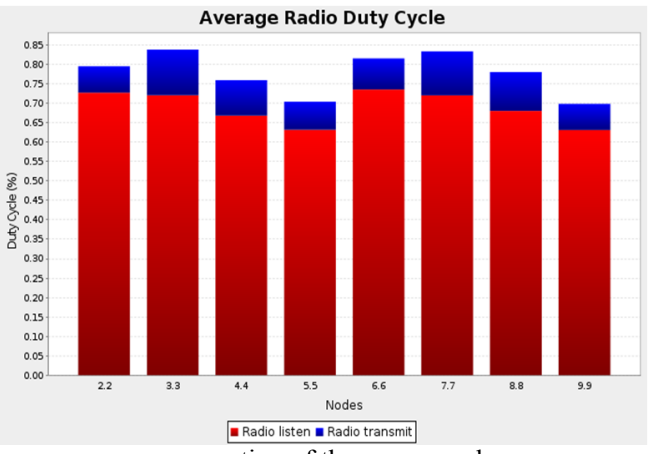

Fig. 9. Average power consumption of the source nodes

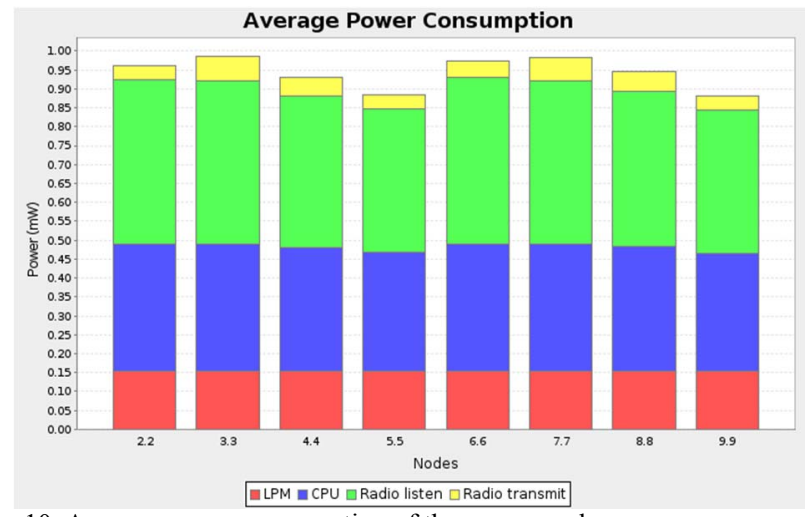

Fig. 10. Average power consumption of the source nodes

\section{B. Packet loss}

The ping ICMP request sent by the client computer in the simulation scenario, which are not acknowledges within the specific waiting time determines the packet loss. Packet loss occurs in wsn networks due to noise, interference and collisions. The scenario is simulated in Cooja by considering TX with $100 \%$ and RX with $90 \%$. The statistics of packet loss is obtained from the reply of ping ICMP packets. Fig. 2 illustrates the packet loss for the scenario. It is seen that the packet loss increase as the packet size and hop increases.

\section{Energy consumption}

In this section, we look at the energy consumption of nodes under the ContikiMAC protocols. We use the duty cycle as an estimator of the energy consumption. The lower the duty cycle, the longer the nodes batteries should last.

ContikiMAC is a suitable and energy efficient radio duty cycle mechanism (RDC) for sensor networks running Contiki. Contiki has a power efficient wake-up mechanism and uses a fast sleep optimization to allow receivers to quickly detect false positive wake-ups. It also uses a transmission optimization to allow run time optimization of the energy efficiency of transmission. The ContikiMAC implementation in Contiki 2.5 uses the Contiki real-time timers (rtimer) to schedule its periodic wake ups. The ContikiMAC wake-up mechanism runs a protothread, which performs the periodic wake, ups and implements the fast sleep optimization. The phase-lock mechanism is implemented as a separate module from ContikiMAC, which maintains a list of neighbors and their wake-up phases. The neighbor is evicted from the list after a fixed number of failed transmissions or having no link layer ack within a fixed time. 
In our experiments, the default parameters of ContikiMAC have been used. Due to the fast sleep optimization the duty-cycle of ContikiMAC is dynamic. The most important contribution to the ContikiMAC reduction in duty-cycle is the fast sleep optimization at the heart of the wake-up procedure. The Duty Cycle provides information regarding the energy consumption of a node by evaluating the time spent by a node in the following states: listen, RX, TX. The Duty Cycle is expressed as the ratio between the time spent by a node in those three states and the wake-up interval. The Duty Cycle value is computed via the Cooja Power tracker tool. The visualizations of radio duty cycle and average power consumption have been collected using the Cooja collect view tool and are presented in Fig. 9 and Fig. 10. From Fig. 8 we observe that the node 5 and 9 experience less duty cycle than other nodes in the network as these are not intermediate node and generate less traffic. Consequently the average power consumption is also less in these two nodes for the same.

\section{CONCLUSION AND FUTURE WORK}

The purpose of this paper is to apply the functionality of Internet of things in the field of agriculture such as smart irrigation to provide optimum use of fresh water and better crops production. The main components of the paper are the system architecture for irrigation control system, the analysis of the performance of the 6LoWPAN protocol stack, Contiki OS and RPL routing protocol in terms of round trip time, packet loss and energy consumption, also investigation of the interoperability between heterogeneous network.

Based on simulation we observe that the RTT and percentage of packet loss will increase as the hop and the payload size become larger due to environmental noise such as interference, collision and link quality. Radio Duty Cycle is a lifetime indicator of the sensors. It is observed from the result that less power consumption means less RDC and hence more lifetime of the sensors. ContikiMAC use a RDC protocol that is responsible for power saving mechanism. The stack simulated in this work is interoperable as it can be connected to different motes platform and does not require gateway to connect to the Internet. Cooja enables emulator with real hardware motes, which make the network simple and reusable. In addition to it, we browse the contents of the sensors from Mozilla browser using Copper plug-in, which implement the CoAP protocol. In a nutshell, one can decide the scalability of WSN in terms of multi hops, positioning of border router, desired latency and data uncertainty in terms of packet loss for real time application of IoT like smart irrigation systems.

In the future we intend to implement this system in a real field scenario so that performance can be analyzed along with the development of web based DSS. There is also an opportunity to analyze the real time data streaming where a lot of data will be transmitted by sensors. We also plan to study how to handle anomalies of data from sensors.

\section{ACKNOWLEDGEMENT}

We would like to extend our gratitude to the Swedish Research Council (under grant 2014-4251) for the funding and the provision of resources to conduct this research.

\section{REFERENCES}

[1] R. Suresh, S. Gopinath, K. Govindaraju, T. Devika and N. Suthanthira Vanitha, "GSM based Automated Irrigation Control using Raingun Irrigation System", International Journal of Advanced Research in Computer and Communication Engineering, Vol. 3, Issue 2, pp. 56545657, 2014.

[2] A. D. Siuli Roy and S. Bandyopadhyay, "Agro-sense: Precision Agriculture using sensor-based wireless mesh networks", Proceedings of the First ITU-T Kaleidoscope Academic Conference, Geneva, Italy, 2008.

[3] R. G. Allen, L. S. Pereira, D. Raes, and M. Smith, Crop Evapotranspiration - Guidelines for Computing Crop Water Requirements-FAO Irrigation and Drainage Paper 56. Rome, Italy:FAO, 1998.

[4] N. R. Patel, P. D. Kale, G. N. Raut, P. G. Choudhari, N. R. Patel, and A. Bherani, "Smart design of microcontroller based monitoring system for agriculture," Proceedings of 2014 International Conference on Circuit, Power and Computing Technologies (ICCPCT), Nagercoil, India, 2014.

[5] J. M. Blonquist Jr., S. B. Jones, and D. A. Robinson, "Precise irrigation scheduling for turfgrass using a subsurface electromagnetic soil moisture sensor," Agricultural Water Management, Vol. 84, Issues 1-2, pp. 153 $165,2006$.

[6] B. Khelifa, D. Amel, B. Amel, C. Mohamed and B. Tarek, "Smart Irrigation Using Internet of Things," Proceedings of the Fourth International Conference on Future Generation Communication Technologies (FGCT 2015), Luton, UK, 2015.

[7] O. M. Grant, M. J. Davies, H. Longbottom, and C. J. Atkinson, "Irrigation scheduling and irrigation systems: Optimising irrigation efficiency for container ornamental shrubs," Irrigation Science, Vol. 27, No. 2, pp. 139-153, 2009.

[8] X. Ma and W. Luo, "The analysis of 6LowPAN technology," Proceedings of Pacific-Asia Workshop on Computational Intelligence and Industrial Application (PACIIA 08), Wuhan, China, 2008.

[9] T. Winter (Ed.), P. Thubert (Ed.), A. Brandt, J. Hui, R. Kelsey, P. Levis, K. Pister, R. Struik, JP. Vasseur, and R. Alexander, "RPL: IPv6 Routing Protocol for Low-Power and Lossy Networks, “ IETF RFC6550, 2012.

[10] N. Accettura, L. A. Grieco, G. Boggia, and P. Camarda, "Performance analysis of the RPL routing protocol," Proceedings of the 2011 IEEE International Conference on Mechatronics, Istanbul, Turkey, 2011.

[11] D. Airehrour, J. Gutierrez and S. Kumar Ray, "Secure routing for internet of things: A survey", Journal of Network and Computer Applications, Vol. 66, pp. 198-213, 2016.

[12] Z. Shelby, K. Hartke, and C. Bormann, "The Constrained Application Protocol (CoAP)," IETF RFC7252, 2014.

[13] Contiki Operating System. http://www.sics.se/contiki/.

[14] S. Thombre, R. Ul Islam, K. Andersson, and M. S. Hossain, "Performance Analysis of an IP based Protocol Stack for WSNs," Proceedings of the 2016 IEEE Conference on Computer Communications Workshops (INFOCOM WKSHPS), 2016.

[15] S. Thombre, R. Ul Islam, K. Andersson, and M. S. Hossain, "IP based Wireless Sensor Networks: Performance Analysis using Simulations and Experiments," Journal of Wireless Mobile Networks, Ubiquitous Computing, and Dependable Applications (JoWUA) 7(3):53-76, 2016. 